\title{
Flow cytometry analysis in atypical aggressive NK leukemia/lymphoma in a 44 year old Caucasian male: A case report
}

Vlad-Andrei Cianga ${ }^{1,2}$, Anne-Camille Faure ${ }^{3}$, Jérémie Stagnara ${ }^{3}$, Lauren Rigollet ${ }^{3}$, Pascale Flandrin-Gresta ${ }^{3}$, Jérôme Cornillon ${ }^{4}$, Denis Guyotat $^{4}$, Lydia Campos Catafal ${ }^{3}$ and Carmen-Mariana Aanei ${ }^{3 *}$

${ }^{1}$ Grigore T. Popa University of Medicine and Pharmacy. Iași, Romania

${ }^{2}$ Department of Clinical Hematology, Regional Institute of Oncology Iasi, Romania

${ }^{3}$ Laboratoire d'Hématologie, Centre Hospitalier Universitaire de Saint-Etienne, Saint-Etienne, France

${ }^{4}$ Institut de cancérologie Lucien-Neuwirth, Saint Priest en Jarez, France

\begin{abstract}
Aggressive Natural Killer cell leukemias/lymphomas (ANKL) are extremely rare lymphoproliferative disorders known for their rapid clinical course, severe symptomatology and poor prognosis. Most cases reported in literature are young Asian adults with a history of Epstein-Barr (EB) infection. Diagnosis is difficult because, there are no molecular makers to assess NK clonality and it relies on clinical, morphological and immunophenotypical evaluation. Here we present the atypical case of a 44-year-old Caucasian male that was diagnosed with ANKL during follow up analysis for hairy cell leukemia (HCL) and we showcase the importance flow cytometry (FCM) had in identifying the neoplastic cell and demonstrating its clonality with the help of killer immunoglobulin-like receptors (KIR).
\end{abstract}

Abbrevations: NK: neoplasms; ANKL: Aggressive Natural Killer cell leukemia/lymphoma; FCM: flow cytometry; KIR: killer immunoglobulin-like receptors

\section{Introduction}

Natural killer (NK) cells neoplasms are a rare and heterogeneous group of lymphoproliferative disorders that are characterized by systemic clonal expansion of NK cells and can involve any organ [1]. According to the 2008 WHO classification of Tumours of Haematopoietic and Lymphoid Tissues, NK neoplasms are represented by the chronic lymphoproliferative disorder of NK cells (CLPD-NK), extranodal NK/ T-cell lymphoma, nasal type (ENKTL) and the aggressive NK leukemia/ lymphoma (ANKL) [2]. ANKL is extremely rare (less than $0,1 \%$ of all lymphoid malignancies) and known for its prevalence among the Asian population, its association with Epstein-Barr virus (EBV) infection and abrupt clinical onset with rapid evolution and poor response to chemotherapy [3-5]. In Europe, such cases are sporadic and have a poor prognosis, with a median survival time of less than 2 months [6].

\section{Case report}

Here we present the case of a 44-year-old Caucasian male diagnosed with ANKL at CHU Saint Etienne, France, in June 2020. This patient has a long and intricate medical record. In 2015, following investigations for splenomegaly, he was diagnosed with hairy cell leukemia (HCL) for which he received treatment with cladribine. Hemolytic anemia and relapse of the disease were discovered in 2018 and Rituximab was added in the therapeutic protocol, resulting in clinical remission. Since March 2020, complete blood count (CBC) showed progressive falls in hemoglobin level and thrombocytopenia, therefore follow-up minimal for residual disease (MRD) was performed in June 2020. Blood tests revealed macrocytic anemia $(\mathrm{Hb}=12 \mathrm{~g} / \mathrm{dl}, \mathrm{MCV}=102 \mathrm{fL})$ with marked reticulocytosis $(\mathrm{RET}=272,000 / \mu \mathrm{l})$, moderate thrombocytopenia $(\mathrm{PLT}=71,000 / \mu \mathrm{l})$, leucopenia $(\mathrm{WBC}=2,100 / \mu \mathrm{l})$ with lymphopenia (lymphocytes $=280 / \mu \mathrm{l})$ and elevated lactate dehydrogenase $(\mathrm{LDH}=644$ UI/l; reference interval 135-250 UI/l). The peripheral blood smear showed persistence of $2 \%$ hairy cells, but also $4.7 \%$ cells with immature blast appearance, hyperbasophilic cytoplasm, low nuclear-cytoplasmic ratio, irregular nuclear outline and weakly condensed chromatin, which raised suspicion of therapy-related acute leukemia (Figure 1).

Bone marrow cytology described a large infiltration of these blast cells (22\%) which often appeared grouped in clusters (Figure 2).

Flow cytometry (FCM) was performed and revealed 3\% residual HCL leukemic cells as well as $80 \%$ abnormal lymphocytes with the sCD3cCD3-CD4-CD8-TCR $\beta \beta-C D 2+C D 7-C D 56+C D 94+C D 16+C D 57-$ $\mathrm{CD} 45+$ phenotype (Figure 3 )

Lymphocytes were gated using light forward scatter (FSC)/linear side scatter (SSC) and CD45/SSC dot plots. Absence of CD19 antigen excluded normal B (Figure 3, dark blue) and residual hairy cells (Figure 3, light blue). T lymphocyte (Figure 3, dark green) markers (sCD3,

${ }^{*}$ Correspondence to: Carmen-Mariana Aanei, Laboratoire d'Hématologie, Centre Hospitalier Universitaire de Saint-Etienne, Saint-Etienne, France, Tel: +33-04-77-82-85-92; E-mail: cmaanei@gmail.com

Key words: neoplasms, aggressive Natural Killer cell leukemia/lymphoma, flow cytometry, EBV negative, killer immunoglobulin-like receptors

Received: September 02, 2020; Accepted: September 11, 2020; Published: September 14, 2020 
cCD3, TCR $\alpha \beta$ ) were also absent and positivity for CD56 and CD16 pointed towards the NK lineage (Figure 4, light green). However, CD7 absence suggested their atypical phenotype. Furthermore, two NK subpopulations could be distinguished: a population was CD56+CD94+CD16+CD57- that accounted for $99.1 \%$ of the total NKs (Figure 4, light green) and a much smaller CD56+CD94+CD16+CD57+ population (0.9\%) (Figure 4).

The predominant CD57- NK population (Figure 5, light green) displayed a strong positivity for all investigated Killer Immunoglobulinlike Receptors (KIR). CD158a was positive in 91\%, CD158b in 97\%, CD158e1 in 99\%, while CD159a (natural killer group 2 receptor) was negative in $97 \%$ of these cells, which points towards an oligoclonal pattern of expression. The minority CD57+ NK cells had however a more heterogeneous receptor distribution, showing only $45 \%$ positivity for CD158a, $46 \%$ positivity for CD158b, $40 \%$ for CD158e1 and $55 \%$ positivity for CD159a (Figure 5).

The immature morphology and bone marrow infiltration, together with the immunophenotypic evidence of oligoclonal NK cells, led to the diagnosis of ANKL. Molecular biology identified BRAF V600E mutation, which is consistent with the previous HCL diagnosis, but otherwise, TCR genes were in germline configuration. The cytogenetic analysis showed a normal karyotype 46, XY [20]. Viral tests were found negative for the following: hepatitis $\mathrm{C}$, cytomegalovirus, parvovirus B19 and EBV. Systemic symptoms, hemophagocytic syndrome, disseminated intravascular coagulation (DIC) or manifestations of organ failure were not apparent at diagnosis.

\section{Discussion}

Literature data offers limited information regarding ANKL due to its rare appearance and, therefore, possibilities to study the disease. We present the atypical case of a Caucasian male diagnosed with ANKL in University Hospital of Saint Etienne. To our knowledge, no other cases where ANKL appears as a secondary lymphoid neoplasm were reported. The disease usually has an extremely aggressive clinical course and constitutional symptoms (fever, night sweats, weight loss), hemophagocytic syndrome or DIC. In our case, the patient did not present typical symptomatology and diagnosis was made in the context of MRD investigation for HCL. Cytology examination and flow cytometry immunophenotyping proved to be indispensable complimentary investigational tools. While morphologically these leukemic cells appeared immature and poorly differentiated, high FSC and SSC parameters and strong expression of CD45, CD16 and KIR surface markers suggest that they, in fact, originate from a phenotypically mature NK cell. Diagnosis of ANKL was difficult due to lack of specific markers that distinguish between reactive and clonal NK cells. In T cell LGLs, sequencing of TCR is used to determine repertoire restriction and clonality, but no such tools are available for NK malignancies [7].

Normal mature NK cells present in the blood and BM can be artificially be divided in 3 subpopulations, which actually represent different stages of NK differentiation [8-10]. The CD56+CD94+CD16subtype is associated with macrophage recruitment and secretion of cytokines and soluble factors (TNFa, IFN $\gamma$ ) [11], while CD56+CD94+/$\mathrm{CD} 16+$ is considered a later stage of development and has mostly cytotoxic properties [12]. Expression of CD57 is associated with loss of proliferation capabilities and cellular memory $[8,13]$. Some studies agree that most ANKL cells have CD56bright/CD16dim/CD57- expression due to differentiation arrest $[4,6,14]$ and clinical manifestations such as hemophagocytic syndrome occur due to the prevalence of the secretory subtype of NK [15]. Hence, we consider the strong expression of CD16 and especially CD57 negativity of the vast majority of the NK cells identified in this patient as markers of a homogeneous malignant population.

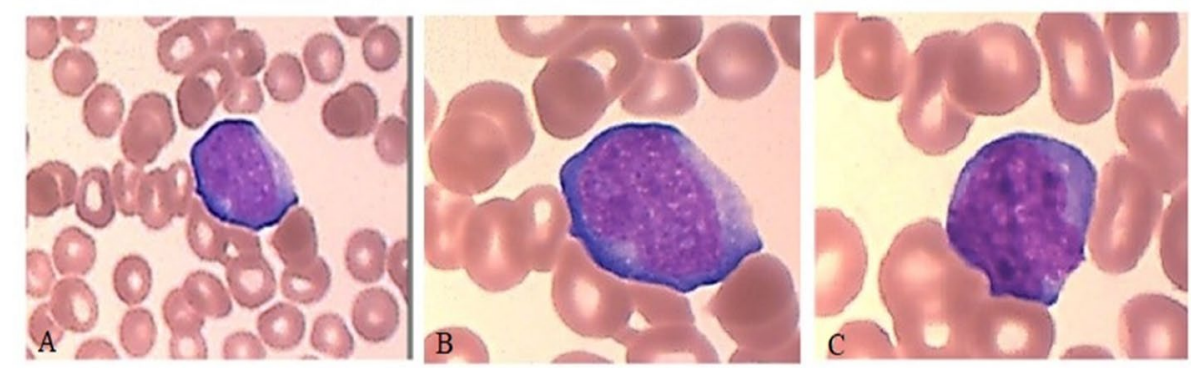

Figure 1. Peripheral blood smear demonstrates a uniform population of immature, hyperbasophilic cells with blast appearance (May-Grünwald-Giemsa staining, 50x (1.A), 100x (1.B, 1.C)
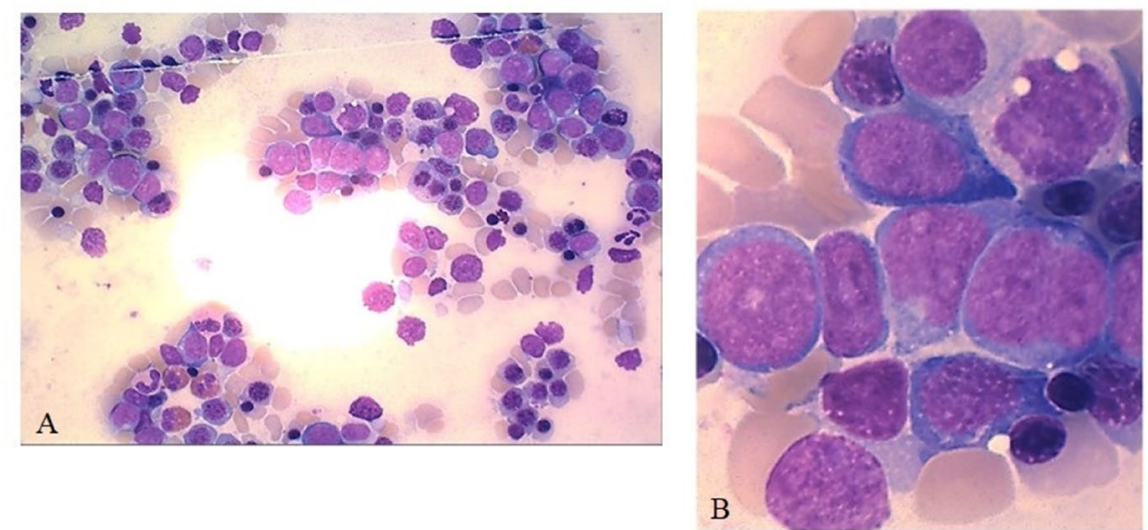

Figure 2. Bone marrow aspirate smear showing involvement by immature, hyperbasophilic cells, 50x (A). Immature blast appearance with low nuclear-cytoplasmic ratio, irregular nuclear outline, weakly condensed chromatin and presence of one or two large nucleoli, 100x (B) 

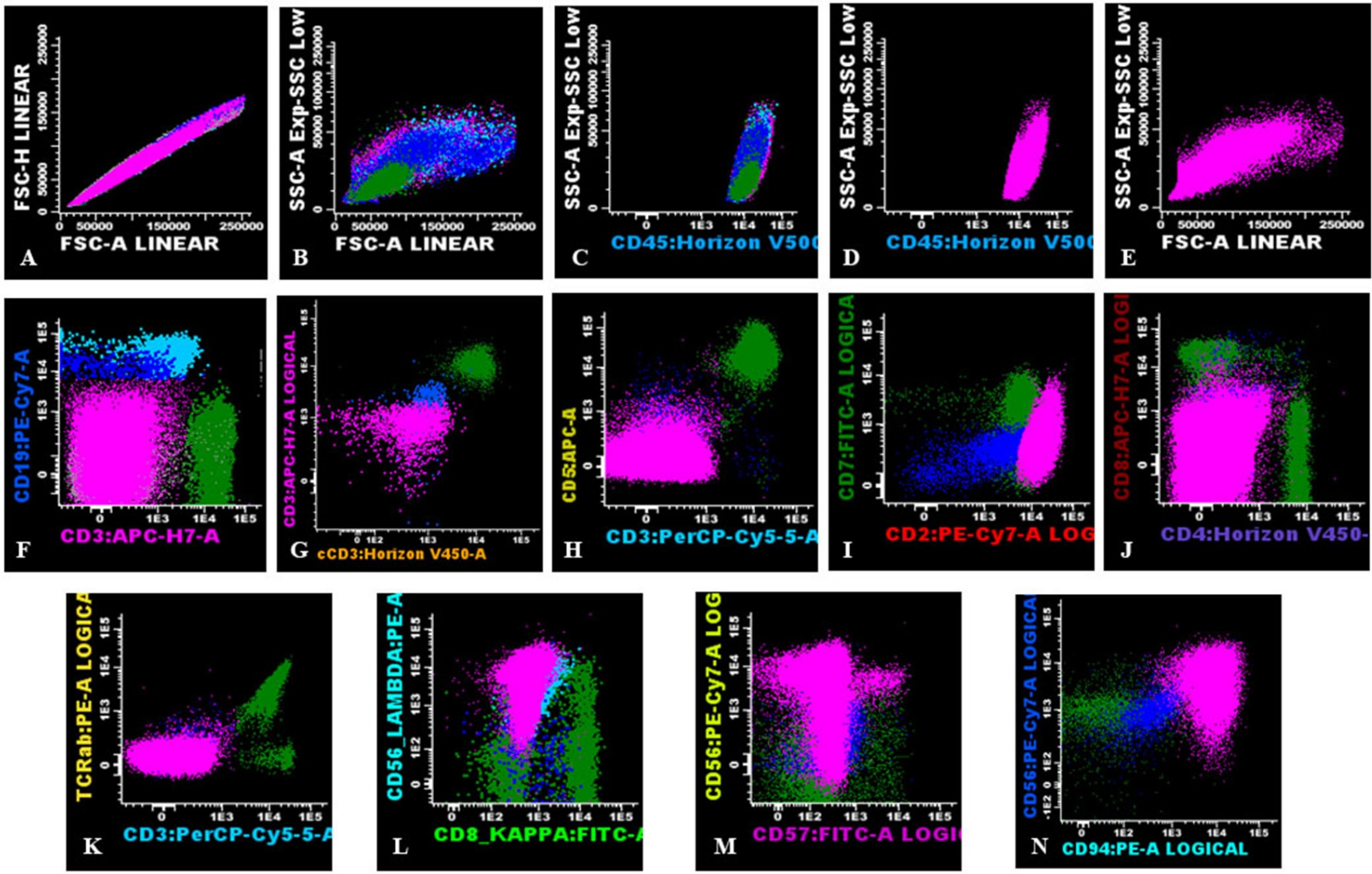

Figure 3. Flow cytometric immunophenotypic findings. An abnormal population is identified (highlighted in pink) with an immunophenotype most consistent with NK cells: increased FSC, SSC (A, B, E), positive for bright CD45 (C), negative for surface and cytoplasmic CD3 (F, G), CD5 (H), CD7 (I), CD4, CD8 (J), surface TCRab (K), and CD57 (M) and, positive for CD2 (I), CD56 (L, M) and, CD94 (N). Dark blue (normal B-cells), light blue (lambda monotypic hairy cells), dark green (normal T cells)
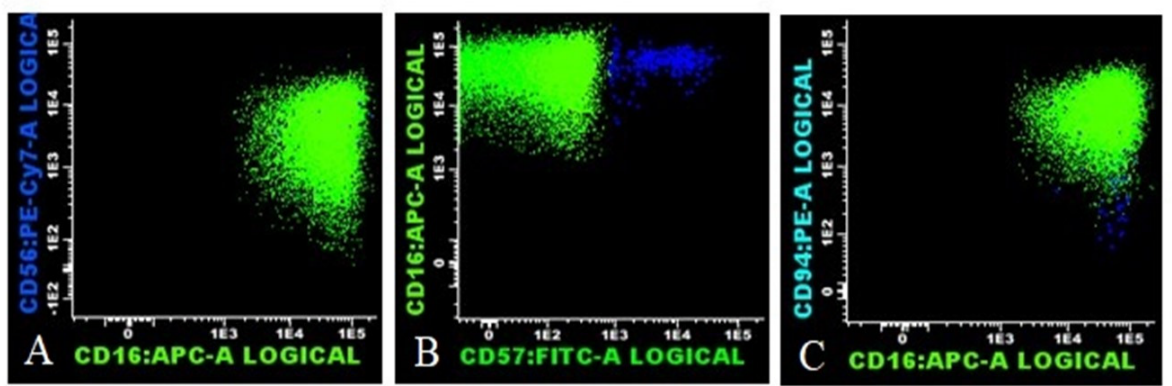

Figure 4. Two distinct subpopulation of NK cells were identified by flow-cytometry, differentiated based on CD57 expression: CD56+CD94+CD16+CD57- NK cells (light green) and CD56+CD94+CD16+CD57+ NK cells (dark blue)
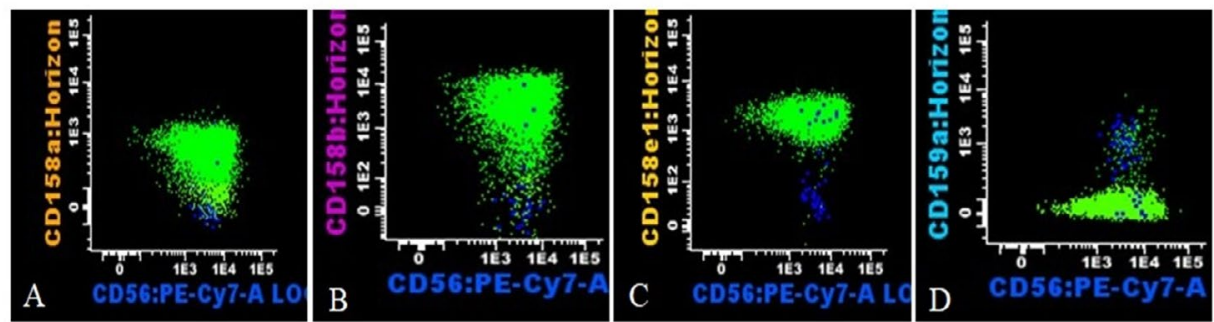

Figure 5. Representative histogram of CD158a, CD158b, CD158e1, and CD159a expression on the NK cell subpopulations (oligoclonal immature NK cells, light green; more mature showing a heterogeneous receptor distribution, dark blue) 
KIRs are extremely polymorphic type 1 transmembrane glycoproteins with Ig-like domains that are generated late during differentiation and are associated with the cytotoxic subtype of NKs $[16,17]$. The NK cells we have characterized in this patient had strong positivity for the CD158a, CD158b, CD158e1 molecules (in $>91 \%$ of leukemic cells), but lacked CD159a expression (97\%), which further argues for the oligoclonality of this NK population. Unlike this subpopulation, the CD57+ NK cells had a more heterogeneous receptor distribution, with positivity ranging between $40-45 \%$, suggestive for a polyclonal character.

Contrary to our findings, other ANKL related studies reported in their investigated cohorts either negativity for CD158a and CD158e1 [4], or a restricted expression of KIR isoforms, even a complete lack of it [6]. While Lima, et al. mention CD159a positivity in twelve analyzed cases ${ }^{4}$, Li's group reports that negativity for $\mathrm{CD} 159$ a was associated with $\mathrm{EBV}$ negative subjects, which is also consistent with our EBV test result ( 0 copies $/ \mathrm{ml})$.

Available treatments are still limited for patients with ANKL, with no established standard protocols. Therapy was scheduled to start after patient had offered written consent and would consist of 2 standard 28 day cycles of SMILE protocol (dexamethasone, methotrexate, ifosfamide, etoposide, and L-asparaginase) followed by potential allogenic hematopoietic stem cell transplant (HSCT). Most experts agree that l-asparaginase based regimens yield better outcomes, however overall survival (OS) is still poor even if they achieve complete remission (CR) $[5,8]$. The introduction of allogenic HSCT after l-asparaginase based chemotherapy showed improved outcomes and OS ( $>1$ year) in a series of selected cases [18-20] and it could play an important role in future therapeutic options. It will be interesting to observe if absence of symptoms at diagnosis and the particular phenotype of the malignant NK will reflect upon the response to therapy and overall survival (OS).

\section{Conclusion}

ANKLs are very rare lymphoid neoplasms, with rapid clinical courses and poor survival rates. Our case is even more particular due to the fact that ANKL is the second hematological malignancy in this patient, with no clinical manifestations or EBV infection at diagnosis. FCM is a sensitive and indispensable tool in identifying leukemic NK cells and showed, in this patient, an aberrant phenotype with absence of CD7, strong positivity for CD16 and homogeneous expression of KIR surface markers. Due to the sporadic appearance of this malignancy, especially in Europeans, available data is limited and there is still much potential for refining diagnostic approaches as well as improving therapeutic management and quality of life for these particular patients.

\section{Consent}

Written consent was obtained for the patient for publication of this case report.

\section{Competing interests}

Authors declare that they do not have competing interests.

\section{Acknowledgments}

We thank Dr. Petru Cianga for suggestions and for the critical reading of this manuscript.

\section{References}

1. Loughran TP, Kadin ME, Starkebaum G (2017) Leukemia of Large Granular Lymphocytes : Association with Clonal Chromosomal Abnormalities and Autoimmune Neutropenia , Thrombocytopenia , and Hemolytic Anemia. Ann Intern Med 102: 169175. [Crossref]

2. Campo E, Swerdlow SH, Harris NL (2014) The 2008 WHO classification of lymphoid neoplasms and beyond: evolving concepts and practical applications. Blood 117: 50195032. [Crossref]

3. Ishida F (2018) Aggressive NK-Cell Leukemia. Pediatric Immunology 6: 1-5.

4. Lima M, Sp A, Fonseca S (2014) Aggressive mature natural killer cell neoplasms: report on a series of 12 European patients with emphasis on fl ow cytometry based immunophenotype and DNA content of neoplastic natural killer cells. Leukemia \& Lymphoma 56: 1-10.

5. Suzuki R (2010) Treatment of advanced extranodal NK/T cell lymphoma, nasal-type and aggressive NK-cell leukemia. Int J Hematol 92: 697-701. [Crossref]

6. Li Y, Wei J, Mao X (2016) Flow Cytometric Immunophenotyping Is Sensitive for the Early Diagnosis of De Novo Aggressive Natural Killer Cell Leukemia (ANKL): A Multicenter Retrospective Analysis. PLoS One 11: e0158827. [Crossref]

7. Lamy T, Moignet A, Jr TPL (2017) LGL leukemia: from pathogenesis to treatment. Blood 129: 1082-1094. [Crossref]

8. Lopez-verge S, Milush JM, Pandey S (2010) CD57 defines a functionally distinct population of mature NK cells in the human CD56 dim CD16 2 NK-cell subset. Blood 116: 3865-3875. [Crossref]

9. Ferlazzo G, Thomas D, Lin S (2015) The Abundant NK Cells in Human Secondary Lymphoid Tissues Require Activation to Express Killer Cell Ig-Like Receptors and Become Cytolytic. J Immunol 172: 1455-1462.

10. Björkström NK, Riese P, Heuts F (2012) Expression patterns of NKG2A, KIR, and CD57 define a process of CD56 dim NK-cell differentiation uncoupled from NK-cell education. Blood 116: 3853-3864. [Crossref]

11. Huntington ND, Vosshenrich CAJ, Santo JP Di (2007) Developmental pathways that generate natural-killer-cell diversity in mice and humans. Nat Rev Immunol 7: 703-714. [Crossref]

12. Zimmer J. Human CD56 dim CD16 dim Cells As an Individualized Natural Killer Cell Subset. Front Immunol 8: 699. [Crossref]

13. Sun JC, Lopez-Verges S, Kim CC, DeRisi JL, Lanier LL (2011) NK Cells and Immune "Memory." J Immunol 186: 1891-1897.

14. Jiang N, Jin Y, Niu Q (2013) Flow cytometric immunophenotyping is of great value to diagnosis of natural killer cell neoplasms involving bone marrow and peripheral blood. Ann Hematol 89-96. [Crossref]

15. Fu B, Tian Z (1983) Subsets of human natural killer cells and their regulatory effects. Immunology 141: 483-489. [Crossref]

16. Pegram HJ, Andrews DM, Smyth MJ, Darcy PK, Kershaw MH (2010) Activating and inhibitory receptors of natural killer cells. Immunol Cell Biol 89: 216-224. [Crossref

17. Del Zotto G, Marcenaro E, Vacca P (2017) Markers and function of human NK cells in normal and pathological conditions. Cytom Part B - Clin Cytom 92: 100-114. [Crossref]

18. Jung KS, Cho S, Kim SJ, Ko YH, Kang E, et al. (2016) L-asparaginase-based regimens followed by allogeneic hematopoietic stem cell transplantation improve outcomes in aggressive natural killer cell leukemia. J Hematol Oncol 9: 41. [Crossref]

19. 19. Ito $\mathrm{T}$, Makishima $\mathrm{H}$, Nakazawa $\mathrm{H}$, et al. Promising approach for aggressive NK cell leukaemia with allogeneic haematopoietic cell transplantation. Eur J Haematol 81: 107-111. [Crossref]

20. 20. Ishida F, Ko YH, Kim WS (2012) Aggressive natural killer cell leukemia Therapeutic potential of 1-asparaginase and allogeneic hematopoietic stem cell transplantation. Cancer Sci 103: 1079-1083. [Crossref]

Copyright: C2020 Cianga V-A. This is an open-access article distributed under the terms of the Creative Commons Attribution License, which permits unrestricted use, distribution, and reproduction in any medium, provided the original author and source are credited. 